\title{
BMJ Open Incidence of surgical site infection following caesarean section: a systematic review and meta-analysis protocol
}

\author{
Khalid B M Saeed, ${ }^{1}$ Richard A Greene, ${ }^{2,3}$ Paul Corcoran, ${ }^{3}$ Sinéad M O'Neill ${ }^{4}$
}

To cite: Saeed KBM, Greene RA, Corcoran P, et al. Incidence of surgical site infection following caesarean section: a systematic review and meta-analysis protocol. BMJ Open 2017;7: 013037 doi:10.1136/bmjopen-2016013037

- Prepublication history and additional material is available. To view please visit the journal (http://dx.doi.org/ 10.1136/bmjopen-2016013037).

Received 14 June 2016 Revised 6 September 2016 Accepted 12 October 2016

CrossMark

\begin{abstract}
${ }^{1}$ Department of Obstetrics and Gynaecology, University College Cork, Cork, Ireland ${ }^{2}$ Cork University Maternity Hospital, Cork, Ireland ${ }^{3}$ Departments of Obstetrics and Gynaecology, National Perinatal Epidemiology Centre, University College Cork, Cork, Ireland ${ }^{4}$ INFANT: Irish Centre for Fetal and Neonatal Translational Research, Cork University Maternity Hospital, Cork, Ireland
\end{abstract}

\section{Correspondence to} Dr Khalid Balla Mohammed Saeed; dr.khalidballa@gmail. com

\section{ABSTRACT}

Introduction: Caesarean section (CS) rates have increased globally during the past three decades. Surgical site infection (SSI) following CS is a common cause of morbidity with reported rates of $3-15 \%$. SSI represents a substantial burden to the health system including increased length of hospitalisation and costs of postdischarge care. The definition of SSI varies with the postoperative follow-up period among different health systems, resulting in differences in the reporting of SSI incidence. We propose to conduct the first systematic review and meta-analysis to determine the pooled estimate for the overall incidence of SSI following CS.

Methods and analysis: We will perform a comprehensive search to identify all potentially relevant published studies on the incidence of SSI following CS reported from 1992 in the English language. Electronic databases including PubMed, CINAHL, EMBASE and Scopus will be searched using a detailed search strategy. Following study selection, full-text paper retrieval, data extraction and synthesis, we will appraise study quality and risk of bias and assess heterogeneity. Incidence data will be combined where feasible in a meta-analysis using Stata software and fixed-effects or random-effects models as appropriate. This systematic review will be reported according to the Preferred Reporting Items for Systematic Reviews and Meta-Analyses (PRISMA) guidelines.

Ethics and dissemination: Ethical approval is not required as this review will use published data. The review will evaluate the overall incidence of SSI following CS and will provide the first quantitative estimate of the magnitude of SSI. It will serve as a benchmark for future studies, identify research gaps and remaining challenges, and emphasise the need for appropriate prevention and control measures for SSI post-CS. A manuscript reporting the results of the systematic review and metaanalysis will be submitted to a peer-reviewed journal and presented at scientific conferences.

Trial registration number: CRD42015024426.

\section{INTRODUCTION}

\section{Rising caesarean section rates}

Globally, caesarean section (CS) rates have increased exponentially over the past three

\section{Strengths and limitations of this study}

- Caesarean section (CS) rates are increasing worldwide and this protocol outlines the plan for a full systematic review and meta-analysis to provide an overall estimate of the incidence of post-CS surgical site infection (SSI) which is currently unknown.

- The proposed systematic review and meta-analysis will adhere to the Preferred Reporting Items for Systematic Reviews and Meta Analyses (PRISMA) guidelines, ensuring consistency and uniformity in reporting and the full systematic review.

- The proposed systematic review and meta-analysis will provide the first estimate of SSI (incisional and organ/space) following CS, using robust and validated Centers for Disease Control (CDC) criteria.

- Two reviewers will screen for study eligibility and perform the quality assessment, minimising the likelihood of reviewer-based bias in the systematic review.

- A limitation of the review is that it will only include the published literature in the English language.

decades. ${ }^{1}$ In the USA, CS was the most commonly performed major surgery in hospitals in 2010 following a $41 \%$ increase in incidence in a 13-year period. ${ }^{2}$ Similarly, high CS rates have been reported in the UK and Australia, where $26.5 \%$ and $32.3 \%$ of births are by CS, respectively. ${ }^{3}{ }^{4}$ In China, the most recent CS rate reported was $41 \% .^{1}$ There are a multitude of driving forces behind the increased rates of CS, including maternal request for CS without medical indication, ${ }^{56}$ as well as fear of litigation among healthcare professionals. ${ }^{7}$ In addition, advancing maternal age may also be contributing to this upward trajectory. ${ }^{8}$

\section{Surgical site infection following caesarean} section

Surgical site infection (SSI) is a term coined by 'The Surgical Wound Infection Task Force' 
in 1992. ${ }^{9}$ The functional definition is the infection, which targets the surgical wound and the uterus when manipulated. ${ }^{910}$ There are precise criteria to define SSI and endometritis for surveillance purposes. ${ }^{9}{ }^{11}$ However, other valid variations have been used. ${ }^{12}$ The incidence of SSI following CS differs with the various methods used for definitions, follow-up tools and time scale of postnatal follow-up. ${ }^{13}$ Independent risk factors are not well documented in the literature. ${ }^{14}$ In a systematic review of the maternal intrinsic risk factors associated with SSI following CS, ${ }^{15}$ obesity and chorioamnionitis were concluded to be common risk factors for the overall SSI, that is, incisional and organ/space together. Ethnicity is debatable as a risk factor for incisional SSI, ${ }^{16}{ }^{17}$ as it is believed to be confounded by other risk factors like obesity, unhealthy diet during pregnancy and socioeconomic status. ${ }^{18}$ In a recent publication, ${ }^{19}$ however, ethnic minority remained a valid risk factor for incisional and organ/space SSI even after multivariable logistic regression. Other factors for overall SSI-incisional and organ/space are: lack or improper use of preoperative prophylaxis antibiotics, ${ }^{20-22}$ labour and chorioamnionitis, ${ }^{23}$ duration of rupture of membranes, emergency CS, and CS for fetal distress. ${ }^{20}$ Suboptimal presurgical haemoglobin and longer duration of surgery also carry a higher risk for overall SSI. ${ }^{21}$

SSI is reported to be the most common hospital-associated infection in community hospital settings. ${ }^{24}$ Moreover, in a recent well-designed multicentre study in England, SSI was estimated to be just under $10 \%$ and the readmission rate due to SSI following CS was $0.6 \%{ }^{25}$ Given that the number of CS deliveries within the UK is $>160000,{ }^{3}$ the cost borne by the health systems will be immense. For example, SSI following CS increases the average length of stay after CS threefold, ${ }^{26}$ which in turn will be reflected in the healthcare cost incurred. In addition, there is the burden on the woman, her family and primary healthcare in the community.

\section{Rationale for current systematic review}

The rate of delivery by CS is increasing globally and the incidence of SSI following CS is likely to show a similar trend; however, a knowledge gap remains on the overall incidence of SSI. Since the definition of SSI following CS varies between different health systems, there is limited scope for making a valid comparison. We draft this protocol for a systematic review to assess the incidence of SSI following CS using the robust and validated Centers for Disease Control (CDC) criteria ${ }^{9}$ and provide the first estimate of SSI to benchmark for individual countries and health systems in the future.

\section{METHODS AND DESIGN}

This systematic review and meta-analysis will follow the Preferred Reporting Items for Systematic Reviews and Meta Analyses (PRISMA) guidelines. ${ }^{27}$

\section{Objectives}

To conduct a systematic review and meta-analysis to estimate the incidence of SSI following CS in the published literature.

\section{Review question}

This systematic review will address the following research question:

What is the incidence of SSI following CS delivery as reported in the literature published from 1992 to 2016?

\section{Criteria for considering studies for the review Inclusion criteria}

1. We will include randomised controlled trials (RCTs), cross-sectional, case-control or cohort studies reporting the incidence of SSI following CS, or studies with enough data to allow us to compute the estimate.

2. We will include studies published in English only from 1992 (as this is when the current CDC criteria were established) regardless of the country or ethnic background of the study population.

3. Any study which reports using the CDC criteria ${ }^{911}$ for diagnosis of SSI will be eligible. In addition, studies where the case definition for SSI-incisional and organ/space met the CDC/National Healthcare Safety Network (NHSN) criteria, and the maximum follow-up time was 6 weeks or 42 days. ${ }^{28}$

The studies will be eligible based on the reporting of SSI within 42 days of the operative procedure and meeting the CDC/NHSN criteria as follows:

1. A superficial incisional SSI: the study must report at least one of the following criteria:

- Purulent drainage

- Organism isolated

- At least one of the following signs and symptoms of infection-pain or tenderness, localised swelling, redness or heat

- The superficial incision is opened by a surgeon unless the incision is culture negative

- The diagnosis is made by a surgeon or attending physician.

2. Deep incisional SSI: the study must report at least one of the following criteria:

- Purulent drainage

- Incision that spontaneously dehisces or is deliberately opened by a surgeon when the patient has at least one of the following signs or symptoms-fever $\left(>38^{\circ}\right)$, localised pain or tenderness unless the incision is culture negative; abscess or other evidence of infection involving deep incision found on direct examination, during reoperation or histopathology, or radiological examination

- The diagnosis is made by a surgeon or attending physician

3. Organ/space SSI: the study must report one or more of the following criteria:

- The patient has an identified organism cultured from endometrial tissue of fluid obtained during the operation. 
The diagnosis is made by a surgeon or attending physician based on at least two of the following: fever $\left(\geq 38^{\circ} \mathrm{C}\right)$ with no other validated causation, purulent drainage from the uterus, abdominal pain or uterine tenderness.

\section{Exclusion criteria}

1. Studies in which the diagnosis of SSI is not based on the CDC/NHSN criteria and where the follow-up extends beyond 42 days.

2. Studies which are not in English.

3. Studies where the participants are not human.

4. Case reports, case-series, letters, commentaries, notes, editorials and conference abstracts.

5. Studies were conducted among a very select group of patients (eg, HIV patients) as they would not be generalisable to the entire population and more susceptible to infection.

6. Whenever multiple publications of the same data exist, we will use the most inclusive, comprehensive and recent one.

\section{Search strategy for identifying relevant studies} Bibliographic database searches

A. We will perform a comprehensive search to identify relevant studies published in English between January 1992 and October 2016. A systematic search of PubMed, EMBASE, CINAHL and Scopus will be performed using a predefined search strategy developed from a combination of the relevant words (eg, 'SSI AND CS'). We will perform the search according to the principles of Boolean logic and incorporate Medical Subject Headings/Entree terms, text words and different versions of spelling of medical terminology (eg, 'caesarean' vs 'cesarean'). The full search strategy is included in online supplementary appendix S1.

B. We will supplement our database searches by manually searching the reference lists of all included papers and relevant reviews.

\section{Selection of studies for inclusion in the review}

One reviewer (KBMS) will undertake the task of running the search strategy across relevant databases and compile the list of retrieved titles in Endnote reference manager. Two investigators (KBMS and SMON) will then independently assess articles by screening titles and abstracts for eligibility. The full text of all studies deemed relevant after the initial screening will be retrieved. Two reviewers (KBMS and RAG) will independently screen the full texts for eligibility and consensus will be obtained by a third reviewer (PC or SMON) where disagreement occurs.

\section{Data extraction and management}

Data will be extracted using a standardised data collection form. Two reviewers will independently extract data including authors, year, country, study design, setting, sample size and diagnosis criteria for SSI used (CDC or other). Where the incidence is not reported directly, we will calculate the incidence using the sample size and number of outcomes. Where these data are missing, contact with the author will be made to request the missing data.

\section{Appraisal of the quality of included studies}

Two reviewers will examine the quality of the included studies. Risk of bias will be judged incorporating the guidelines set out in the Cochrane Handbook for Systematic Reviews of Interventions when assessing RCTs $^{29}$ For observational studies deemed eligible for inclusion, we will use the quality assessment tool for quantitative studies developed by the Effective Public Health Practice Project (EPHPP), Ontario, Canada, which has been evaluated in a previous study. ${ }^{30}$ The quality of each study will be scored and a subgroup analysis performed according to high-quality and low-quality studies in the meta-analysis.

\section{Data synthesis including assessment of heterogeneity}

Incidence data will be summarised using STATA software V.14 (STATA Corporation, Texas, USA) to perform a meta-analysis and generate a pooled estimate of the overall incidence of SSI following CS. Where heterogeneity is $>50 \%$ (based on the $\mathrm{I}^{2}$ statistic, ${ }^{31}$ we will use the random-effects model. We will assess the presence of publication bias using a funnel plot and Egger's test ${ }^{32}$ (provided we have at least 10 studies included in the meta-analysis). Where heterogeneity is present, we will attempt to explain it with subgroup analyses including: by definition of SSI used CDC or other (modified CDC with duration of follow-up at a maximum of 42 days $^{28}$ ); setting (high-income countries vs low-income or middle-income countries); study quality (high vs low); study design (case-control, cohort, cross-sectional); type of SSI (incisional or organ/space); length of SSI follow-up ( $<1$ week, $>1$ week $<30$ days, $>30$ days $<42$ days), methods of reporting SSI (patient reported (eg, telephone or questionnaire) vs health personnel (public health nurse, general practitioner in the community, etc)) and time period (pre-2000, post-2000).

\section{PRESENTING AND REPORTING THE RESULTS}

We will use a flow diagram to summarise the study selection process and show the excluded articles and rationale for exclusion. The characteristics and quality assessment of the included studies will be presented in tables. Quantitative estimates of the incidence of SSI will be presented in a forest plot or individually in tables as appropriate.

\section{CONCLUSIONS}

CS rates have reached their highest levels until now accounting for half of all deliveries in some countries. With this, SSIs are increasing and there is an increased cost burden on the healthcare systems as well as 
personally to mothers and their families. The first step is to quantify this burden for the first time and estimate the magnitude of the incidence of SSI following CS. This systematic review and meta-analysis will influence policy, practice and future research and empower stakeholders including nurses in the operating theatre to patients in the community. Variations in definitions and diagnostic criteria for SSI have made it difficult for the health authorities in different health systems to benchmark each other and variations in the reporting of SSIs exist as a result. In the future, a standard definition of SSI globally would help to minimise this problem.

We will perform the proposed systematic review and meta-analysis to compute the overall incidence of SSI following CS following a predefined protocol, including a comprehensive search strategy, standardised data extraction, quality appraisal by multiple reviewers and a priori defined subgroup analyses to minimise bias. Possible limitations include the restriction of the inclusion of studies published in English and the quality of the data retrieved from the studies. In conclusion, this review will provide the first estimate of SSI post-CS and identify any gaps in research until now warranting further research. It may also be helpful for maternity units to compare their rates as a predictor of service quality and could provide a base for a wider comparator among different health authorities and jurisdictions.

\section{ETHICS AND DISSEMINATION}

Since this is a protocol for a systematic review and meta-analysis, which will be based on published data and, as such, ethical approval is not required. This study incorporating the available published data on SSI since 1992 will be prepared as a manuscript to be published in a peer-reviewed journal. Furthermore, the results will be disseminated through scientific venues, conferences and among relevant healthcare stakeholders. We aim to update this piece of scientific work in the future to inform policy and health service solutions.

Twitter Follow Sinead 0'Neill @sineadoneill13

Contributors KBMS, RAG and PC conceived the study. KBMS wrote the first draft of this protocol. RAG, PC and SMON revised the manuscript for methodological content. All authors approved the final version.

Funding Department of Obstetrics and Gynaecology, University College Cork, Cork, Ireland.

Competing interests None declared.

Provenance and peer review Not commissioned; externally peer reviewed.

Open Access This is an Open Access article distributed in accordance with the Creative Commons Attribution Non Commercial (CC BY-NC 4.0) license, which permits others to distribute, remix, adapt, build upon this work noncommercially, and license their derivative works on different terms, provided the original work is properly cited and the use is non-commercial. See: http:// creativecommons.org/licenses/by-nc/4.0/

\section{REFERENCES}

1. Betran AP, Merialdi M, Lauer JA, et al. Rates of caesarean section: analysis of global, regional and national estimates. Paediatr Perinat Epidemiol 2007;21:98-113.
2. Pfuntner A, Wier LM, Stocks C. Most Frequent Procedures Performed in U.S. Hospitals, 2010. HCUP Statistical Brief \#149. 2013.

3. Hospital Episode Statistics Analysis HaSCIC. NHS Maternity Statistics-England, 2014-15. http://www.hscic.gov.uk2015

4. Li Z, Zeki R, Hilder L, et al. 2013. Australia's mothers and babies 2011. Perinatal statistics series no. 28. Cat. no. PER 59. Canberra: AlHW National Perinatal Epidemiology and Statistics Unit, 2011.

5. Gossman GL, Joesch JM, Tanfer K. Trends in maternal request caesarean delivery from 1991 to 2004. Obstet Gynecol 2006;108:1506-16.

6. National Institutes of Health state-of-the-science conference statement: cesarean delivery on maternal request March 27-29, 2006. Obstet Gynecol 2006;107:1386-97.

7. Murthy K, Grobman WA, Lee TA, et al. Association between rising professional liability insurance premiums and primary caesarean delivery rates. Obstet Gynecol 2007;110:1264-9.

8. Joseph KS, Young DC, Dodds L, et al. Changes in maternal characteristics and obstetric practice and recent increases in primary caesarean delivery. Obstet Gynecol 2003;102:791-800.

9. Horan TC, Gaynes RP, Martone WJ, et al. CDC definitions of nosocomial surgical site infections, 1992: a modification of CDC definitions of surgical wound infections. Infect Control Hosp Epidemiol 1992;13:606-8.

10. Garner JS, Jarvis WR, Emori TG, et al. CDC definitions for nosocomial infections, 1988. Am J Infect Control 1988;16: $128-40$.

11. Horan TC, Andrus M, Dudeck MA. CDC/NHSN surveillance definition of healthcare-associated infection and criteria for specific types of infections in the acute care setting. Am J Infect Control 2008;36:309-32.

12. Wilson AP, Treasure T, Sturridge MF, et al. A scoring method (ASEPSIS) for postoperative wound infections for use in clinical trials of antibiotic prophylaxis. Lancet 1986;1:311-13.

13. Bruce J, Russell EM, Mollison J, et al. The measurement and monitoring of surgical adverse events. Health Technol Assess 2001:5:1-194.

14. Olsen MA, Butler AM, Willers DM, et al. Risk factors for surgical site infection after low transverse caesarean section. Infect Control Hosp Epidemiol 2008;29:477-84; discussion 85-6.

15. Lakhan P, Doherty J, Jones M, et al. A systematic review of maternal intrinsic risk factors associated with surgical site infection following caesarean sections. Healthc Infect 2010;15:35-41.

16. Beattie PG, Rings TR, Hunter MF, et al. Risk factors for wound infection following caesarean section. Aust N Z J Obstet Gynaecol 1994;34:398-402.

17. Noyes N, Berkeley AS, Freedman K, et al. Incidence of postpartum endomyometritis following single-dose antibiotic prophylaxis with either ampicillin/sulbactam, cefazolin, or cefotetan in high-risk caesarean section patients. Infect Dis Obstet Gynecol 1998;6:220-3

18. Olsen MA, Butler AM, Willers DM, et al. Risk factors for endometritis after low transverse caesarean delivery. Infect Control Hosp Epidemiol 2010;31:69-77.

19. Assawapalanggool S, Kasatpibal N, Sirichotiyakul S, et al. Risk factors for caesarean surgical site infections at a Thai-Myanmar border hospital. Am J Infect Control 2016;44:990-5.

20. Farret TCF, Dallé J, da Silva Monteiro V, et al. Risk factors for surgical site infection following caesarean section in a Brazilian Women's Hospital: a case-control study. Braz J Infect Dis 2015;19:113-17.

21. Gong SP, Guo HX, Zhou HZ, et al. Morbidity and risk factors for surgical site infection following caesarean section in Guangdong Province, China. J Obstet Gynaecol Res 2012;38:509-15.

22. Killian CA, Graffunder EM, Vinciguerra TJ, et al. Risk factors for surgical-site infections following caesarean section. Infect Control Hosp Epidemiol 2001;22:613-17.

23. Shree R, Park SY, Beigi RH, et al. Surgical site infection following cesarean delivery: patient, provider, and procedure-specific risk factors. Am J Perinatol 2016;33:157-64.

24. Lewis SS, Moehring RW, Chen LF, et al. Assessing the relative burden of hospital-acquired infections in a network of community hospitals. Infect Control Hosp Epidemiol 2013:34:1229-30.

25. Wloch C, Wilson J, Lamagni T, et al. Risk factors for surgical site infection following caesarean section in England: results from a multicentre cohort study. BJOG 2012;119:1324-33.

26. Jenks PJ, Laurent M, McQuarry S, et al. Clinical and economic burden of surgical site infection (SSI) and predicted financial consequences of elimination of SSI from an English hospital. $J$ Hosp Infect 2014;86:24-33. 
27. Moher D, Liberati A, Tetzlaff J, et al. Preferred reporting items for systematic reviews and meta-analyses: the PRISMA statement. PLoS Med 2009;6:e1000097.

28. Duggal N, Poddatorri V, Noroozkhani S, et al. Perioperative oxygen supplementation and surgical site infection after caesarean delivery: a randomized trial. Obstet Gynecol 2013;122:79-84.

29. Higgins JPT, Green S. Cochrane Handbook for Systematic Reviews of Interventions Version 5.1.0 [updated March 2011]. The Cochrane Collaboration, 2011. http://www.cochrane-handbook.org (accessed 8 Jun 2016).
30. Armijo-Olivo S, Stiles CR, Hagen NA, et al. Assessment of study quality for systematic reviews: a comparison of the Cochrane Collaboration Risk of Bias Tool and the Effective Public Health Practice Project Quality Assessment Tool: methodological research. J Eval Clin Pract 2012;18:12-18.

31. Higgins JP, Thompson SG. Quantifying heterogeneity in a meta-analysis. Stat Med 2002;21:1539-58.

32. Egger M, Davey Smith G, Schneider M, et al. Bias in meta-analysis detected by a simple, graphical test. BMJ 1997;315:629-34. 